\title{
Floquet second-order topological superconductor driven via ferromagnetic resonance
}

\author{
Kirill Plekhanov [D, Manisha Thakurathi, Daniel Loss, and Jelena Klinovaja \\ Department of Physics, University of Basel, Klingelbergstrasse 82, CH-4056 Basel, Switzerland
}

(Received 23 May 2019; published 1 November 2019)

\begin{abstract}
We consider a Floquet triple-layer setup composed of a two-dimensional electron gas with spin-orbit interactions, proximity coupled to an $s$-wave superconductor and to a ferromagnet driven at resonance. The ferromagnetic layer generates a time-oscillating Zeeman field which competes with the induced superconducting gap and leads to a topological phase transition. The resulting Floquet states support a second-order topological superconducting phase with a pair of localized zero-energy Floquet Majorana corner states. Moreover, the phase diagram comprises a Floquet helical topological superconductor, hosting a Kramers pair of Majorana edge modes protected by an effective time-reversal symmetry, as well as a gapless Floquet Weyl phase. The topological phases are stable against disorder as well as against parameter variations and are within experimental reach.
\end{abstract}

DOI: 10.1103/PhysRevResearch.1.032013

Introduction. Over the last decade topological states of matter [1-5] have attracted a lot of attention. Recently, particular interest has been raised by higher-order topological insulators and superconductors [6-8], which host topologically protected gapless modes on their higher-order faces (e.g., corners in $d>1$, hinges in $d>2$, with $d$ being the spatial dimension of the system). However, such systems were studied mostly at the static level [9-19], with the out-of-equilibrium dynamics [20-27] rarely addressed. At the same time, Floquet engineering [28-31], based on applying time-periodic perturbations, has been serving as a powerful tool to generate exotic phases of quantum matter, including topological and Chern insulators [32-51], as well as non-Abelian states such as Majorana bound states (MBSs) [52-60]. Such Floquet phases can be generated by time-dependent electromagnetic fields [32-39,58,59]. Strong oscillating electric fields are easy to obtain for this purpose, but the direct coupling to the spin degree of freedom is more challenging, typically achieved only indirectly via a strong spin-orbit interaction [61,62]. Hence, finding ways to generate strong oscillating magnetic fields which couple directly to the spin is important. One possible solution consists in using magnetic proximity effects, which, for the static case, have already been widely studied for superconductors [63,64] and topological insulators [65-67].

In this Rapid Communication we consider a driven triplelayer setup which allows us to engineer a Floquet higher-order topological superconductor (FHOTS). Its key component is a two-dimensional electron gas (2DEG) with a spin-orbit interaction (SOI) sandwiched between an $s$-wave superconductor (SC) and a ferromagnet (FM), as shown in Fig. 1(a). The FM layer is resonantly driven by an external field $\boldsymbol{H}(t)$, which results in the generation of an oscillating magnetic field $\boldsymbol{B}(t)$,

Published by the American Physical Society under the terms of the Creative Commons Attribution 4.0 International license. Further distribution of this work must maintain attribution to the author(s) and the published article's title, journal citation, and DOI. giving rise to strong Zeeman coupling in the 2DEG. The out-of-plane component of $\boldsymbol{B}(t)$ competes with the proximityinduced superconducting gap and leads to a topological phase transition in the 2DEG. The topological phase is identified as Floquet helical topological superconductor (FHeTS), characterized by the presence of a Kramers pair of gapless helical Majorana edge modes protected by an effective time-reversal symmetry. A moderate in-plane component of $\boldsymbol{B}(t)$ opens a gap in the helical edge modes through a mass term that changes sign at the corners in a rectangular geometry, resulting in Majorana corner states (MCSs) - a distinct feature of the FHOTS. In the regime where the in-plane component of $\boldsymbol{B}(t)$ is strong, the system moves into a gapless Weyl phase. The topological phase diagram is robust against moderate local disorder, detuning from resonance, and static magnetic fields.

Model. The triple-layer setup is composed of a 2DEG with strong Rashba and Dresselhaus SOIs, proximity coupled to an $s$-wave SC and a FM, as shown in Fig. 1(a). We assume that the SOI vector is along the $z$ direction, which is perpendicular to the $x y$ 2DEG plane. The SOI coupling strengths $\alpha_{x}=\alpha_{R}+\beta_{D}$ and $\alpha_{y}=\alpha_{R}-\beta_{D}$ are expressed in terms of the Rashba and Dresselhaus SOI coefficients $\alpha_{R}$ and $\beta_{D}$ for a proper choice of the coordinate system [68-75]. Introducing a creation operator $\psi_{\sigma k}^{\dagger}$ acting on an electron with momentum $\boldsymbol{k}=\left(k_{x}, k_{y}\right)$ and spin component $\sigma$ along the $z$ axis, the corresponding Hamiltonian reads

$H_{0}=\sum_{\sigma \sigma^{\prime}} \int d \boldsymbol{k} \psi_{\sigma \boldsymbol{k}}^{\dagger}\left[\frac{\hbar^{2} \boldsymbol{k}^{2}}{2 m}-\mu-\alpha_{x} k_{x} \sigma_{y}+\alpha_{y} k_{y} \sigma_{x}\right]_{\sigma \sigma^{\prime}} \psi_{\sigma^{\prime} \boldsymbol{k}}$

Here, $\sigma_{j}$ are the Pauli matrices acting in spin space. The chemical potential $\mu$ is calculated from the crossing point of the two spin-split bands at $\boldsymbol{k}=\mathbf{0}$. In the following, it will be convenient to introduce the SOI energy $E_{\mathrm{so}}=\hbar^{2} k_{\mathrm{so}}^{2} /(2 m)$ and the SOI momentum $k_{\text {so }}=m \alpha_{x} / \hbar^{2}$. We note that this Hamiltonian effectively describes a $2 \mathrm{D}$ array of coupled Rashba wires 


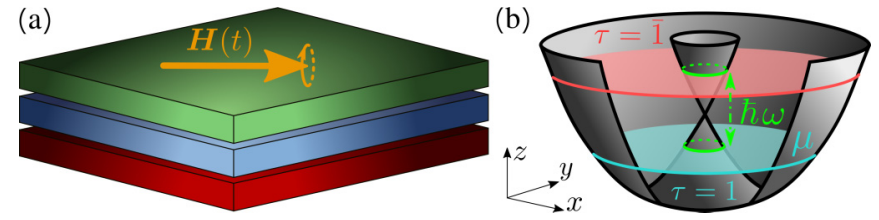

FIG. 1. (a) Schematics of the Floquet triple-layer setup consisting of a 2DEG (blue layer) with SOI of strengths $\alpha_{x}$ and $\alpha_{y}$, proximity coupled to an $s$-wave SC (red layer) at the bottom and a FM at the top (green layer) which is resonantly driven by an external timedependent magnetic field $\boldsymbol{H}(t)$ to generate an oscillating magnetic field $\boldsymbol{B}(t)=B_{\perp} \cos (\omega t) \boldsymbol{e}_{z}+B_{\|} \sin (\omega t) \boldsymbol{u}_{\|}$in the 2DEG. (b) Band structure of the 2DEG in the isotropic regime $\alpha_{x}=\alpha_{y}$. The chemical potential $\mu$ is fixed below the crossing point of the spin-split bands (indicated by the blue area). The driving frequency $\omega$ is tuned to achieve resonance at the smallest Fermi momentum (represented by the two green circles) between the two Floquet bands labeled by $\tau= \pm 1$.

if the mass $m$ is also chosen to be anisotropic in the $x y$ plane such that $m_{y} \neq m_{x}[39,76-87]$.

The proximity effect between the $2 \mathrm{DEG}$ and the $\mathrm{SC}$ is described by the following Hamiltonian,

$$
H_{\mathrm{sc}}=\frac{\Delta_{\mathrm{sc}}}{2} \sum_{\sigma \sigma^{\prime}} \int d \boldsymbol{k}\left(\psi_{\sigma \boldsymbol{k}}^{\dagger}\left[i \sigma_{y}\right]_{\sigma \sigma^{\prime}} \psi_{\sigma^{\prime}(-\boldsymbol{k})}^{\dagger}+\text { H.c. }\right) \text {, }
$$

where $\Delta_{\mathrm{sc}}$ is the induced SC gap. The resulting 2DEG-SC heterostructure is placed in the vicinity of a FM layer, and the setup is subjected to an external magnetic field $\boldsymbol{H}(t)$. Under the FM resonance condition (see the discussion below), the FM generates an oscillating demagnetizing field which adds up to $\boldsymbol{H}(t)$ to produce a total magnetic field $\boldsymbol{B}(t)=$ $B_{\perp} \cos (\omega t) \boldsymbol{e}_{z}+B_{\|} \sin (\omega t) \boldsymbol{u}_{\|}$in the 2DEG. Here, $\omega=2 \pi / T$ denotes the oscillation frequency and the $2 \mathrm{D}$ vector $\boldsymbol{u}_{\|}=$ $\left(u_{x}, u_{y}\right)$ indicates the orientation of the magnetic field in the $x y$ plane. The FM proximity effect is described by the following Floquet-Zeeman term

$$
\begin{aligned}
H_{\mathrm{Z}}(t)= & 2 \sum_{\sigma \sigma^{\prime}} \int d \boldsymbol{k} \psi_{\sigma \boldsymbol{k}}^{\dagger}\left(t_{\mathrm{Z}}^{\perp} \cos (\omega t)\left[\sigma_{z}\right]_{\sigma \sigma^{\prime}}\right. \\
& \left.+t_{\mathrm{Z}}^{\|} \sin (\omega t)\left[\boldsymbol{u}_{\|} \cdot \boldsymbol{\sigma}\right]_{\sigma \sigma^{\prime}}\right) \psi_{\sigma^{\prime} \boldsymbol{k}},
\end{aligned}
$$

where $t_{\mathrm{Z}}^{v}=\mu_{B} g_{v} B_{v} / 2$ (with $v=\|, \perp$ ) are two FloquetZeeman amplitudes. The anisotropy in the $g$-factors, which leads to $g_{\|}$and $g_{\perp}$, arises from the quantum confinement and the intrinsic strain of the 2DEG [88-90].

The resulting time-dependent problem can be solved using the Floquet formalism [28-31], by writing the quasienergy operator $H=H_{0}+H_{\mathrm{sc}}+H_{\mathrm{Z}}(t)-i \hbar \partial_{t}$ in the Floquet-Hilbert space generated by $T$-periodic states $\psi_{l \sigma k}=\exp (-i l \omega t) \psi_{\sigma k}$, $l \in \mathbb{Z}$. In this basis $H$ acquires a simple block-diagonal form, where each block, also referred to as a Floquet band, is composed of the modes with the same index $l$. The static term acts within the same block and receives an additional constant energy shift $\hbar \omega l$, while the oscillating term couples different blocks. We note that, in contrast to static setups [91-94], our protocol does not require a fine tuning of the chemical potential. Instead of tuning $\mu$ close to the spin-orbit energy, $\mu \approx 0$, we only need to require that $-E_{\text {so }}<\mu<0$, so that the frequency $\omega$ can be resonantly tuned to the energy difference between the two spin-split bands. This allows us to treat the oscillating terms at low energies by only taking into account the coupling between the modes at $l=0$, to which we associate a Floquet band index $\tau=1$, and the modes at $l=-1$ with $\tau=\overline{1}$. As a result, in the Nambu basis $\Psi_{k}^{\dagger}=$ $\left(\psi_{1 \uparrow k}^{\dagger}, \psi_{1 \downarrow k}^{\dagger}, \psi_{\overline{1} \uparrow k}^{\dagger}, \psi_{\overline{1} \downarrow k}^{\dagger}, \psi_{1 \uparrow-k}, \psi_{1 \downarrow-k}, \psi_{\overline{1} \uparrow-k}, \psi_{\overline{1} \downarrow-k}\right)$, the total Hamiltonian reads as $H=\int d \boldsymbol{k} \Psi_{\boldsymbol{k}}^{\dagger} \mathcal{H}_{\boldsymbol{k}} \Psi_{\boldsymbol{k}} / 2$, with the Hamiltonian density

$$
\begin{aligned}
\mathcal{H}_{\boldsymbol{k}}= & {\left[\frac{\hbar^{2} \boldsymbol{k}^{2}}{2 m}-\mu+\frac{\hbar \omega\left(\tau_{z}-\tau_{0}\right)}{2}\right] \eta_{z}+\Delta_{\mathrm{sc}} \eta_{y} \sigma_{y}+\alpha_{y} k_{y} \sigma_{x} } \\
& -\alpha_{x} k_{x} \eta_{z} \sigma_{y}+t_{\mathrm{Z}}^{\perp} \eta_{z} \tau_{x} \sigma_{z}+t_{\mathrm{Z}}^{\|}\left(u_{x} \tau_{y} \sigma_{x}+u_{y} \eta_{z} \tau_{y} \sigma_{y}\right)
\end{aligned}
$$

with the Pauli matrices $\tau_{i}\left(\eta_{i}\right)$ acting in the Floquet (particlehole) space. In the following, we analyze different topological phases of the system as a function of the parameters appearing in Eq. (4).

Floquet helical topological superconductor. In order to determine the phase diagram of our model, we first consider the effect of the out-of-plane component of $\boldsymbol{B}(t)$ by imposing $t_{\mathrm{Z}}^{\|}=0$ in Eq. (4). In the isotropic regime with $\alpha_{x}=\alpha_{y}$, the Fermi surface is composed of two concentric circles and the problem depends only on the magnitude of the momentum $|\boldsymbol{k}|$, as shown in Fig. 1(b). The resonance condition for the frequency $\omega$ is satisfied along the entire circle with the smallest Fermi momentum [see the Supplemental Material (SM) [95] for more details]. The Hamiltonian is linearized close to the Fermi surface and provides the eigenenergies $E_{1}^{2}=\left(\hbar v_{F} \delta k\right)^{2}+\Delta_{\mathrm{sc}}^{2}$ and $E_{2, \pm}^{2}=\left(\hbar v_{F} \delta k\right)^{2}+$ $\left(t_{\mathrm{Z}}^{\perp} \pm \Delta_{\mathrm{sc}}\right)^{2}$, with $v_{F}=\alpha_{x} / \hbar$ the Fermi velocity and $\delta k$ the radial distance from the Fermi surface. The phase diagram consists of two gapped phases separated by the gapless line $\Delta_{\mathrm{sc}}=t_{\mathrm{Z}}^{\perp}$. The topologically trivial (topological) phase is identified with the regime $\Delta_{\mathrm{sc}}>t_{\mathrm{Z}}^{\perp}\left(\Delta_{\mathrm{sc}}<t_{\mathrm{Z}}^{\perp}\right)$. For $t_{\mathrm{Z}}^{\|}=0$, the system is characterized by an emerging effective timereversal symmetry $T_{\text {eff }}=-i \tau_{z} \sigma_{y} \mathcal{K}$, a particle-hole symmetry $P=\eta_{x} \mathcal{K}$, and a chiral symmetry $U_{C}=P T_{\text {eff }}$, with $\mathcal{K}$ the complex-conjugation operator. Thus, the system belongs to the DIII symmetry class with $\mathbb{Z}_{2}$ topological invariant.

The topological phase, denoted as FHeTS, hosts gapless edge modes - a Kramers pair of Floquet Majorana fermions $\left|\Phi_{ \pm}\right\rangle$, obeying $P\left|\Phi_{ \pm}\right\rangle=\left|\Phi_{ \pm}\right\rangle$and $T_{\text {eff }}\left|\Phi_{ \pm}\right\rangle= \pm\left|\Phi_{\mp}\right\rangle$. The Kramers partners propagate in opposite directions along the same edge, forming a pair of helical modes protected by the effective time-reversal and particle-hole symmetries. We have verified the presence of these modes numerically in the discretized version of the model (see the SM [95]) defined on a rectangular lattice of size $L_{x} \times L_{y}$ with periodic boundary conditions along the $x$ axis, as shown in Fig. 2(a).

If the rotation symmetry is broken $\left(\alpha_{x} \neq \alpha_{y}\right)$, the resonance condition can be satisfied only along a particular direction in momentum space, resulting in an offset $\delta \omega$ in the resonance condition almost everywhere except at a few points on the Fermi surface. While a small $\delta \omega$ in the weak anisotropy regime hardly affects the phase diagram and can be compensated by increasing $t_{\mathrm{Z}}^{\perp}$, strong anisotropy effects are more drastic. In Fig. 2(b), we calculate numerically the energy of 

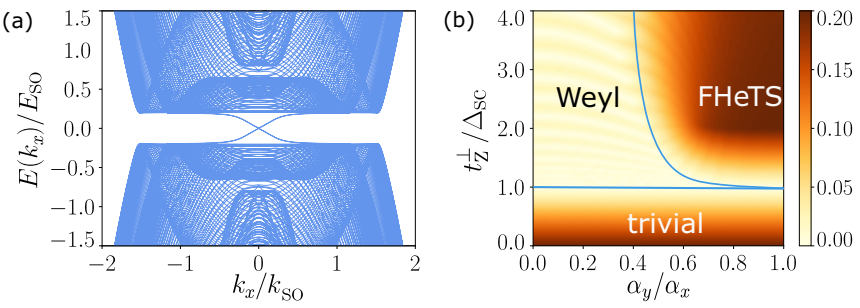

FIG. 2. (a) Energy spectrum $E\left(k_{x}\right)$ in the FHeTS phase with $t_{\mathrm{Z}}^{\perp} / \Delta_{\mathrm{sc}}=3$ and $\alpha_{x}=\alpha_{y}$. The helical edge modes are localized at the edges. (b) Phase diagram showing the gap to the first excited bulk state (in units of $E_{\mathrm{so}}$ ) as a function of the ratios $\alpha_{y} / \alpha_{x}$ and $t_{\mathrm{Z}}^{\perp} / \Delta_{\mathrm{sc}}$. Blue lines indicate the phase boundaries. In the isotropic regime, $\alpha_{x}=\alpha_{y}$, the phase transition occurs at the critical point $\Delta_{\mathrm{sc}}=t_{\mathrm{Z}}^{\perp}$. When $\alpha_{x} \neq \alpha_{y}$, the critical point is transformed into a gapless Weyl phase, and the value $t_{\mathrm{Z}}^{\perp} / \Delta_{\text {sc }}$ required to reach the FHeTS increases up to the point of strong anisotropy beyond which the FHeTS cannot be reached. Remaining parameters in both simulations are $t_{\mathrm{Z}}^{\|}=0$, $\Delta_{\mathrm{sc}} / E_{\mathrm{so}}=0.2, k_{\mathrm{so}} L_{x}=k_{\mathrm{so}} L_{y}=80$, and $\mu=-E_{\mathrm{so}} / 2$.

the lowest bulk state as a function of the ratios $\alpha_{y} / \alpha_{x}$ and $t_{\mathrm{Z}}^{\perp} / \Delta_{\text {sc }}$. We see that the critical point $\Delta_{\mathrm{sc}}=t_{\mathrm{Z}}^{\perp}$ transforms into a gapless Weyl phase at a finite value of anisotropy $\left(\alpha_{y}-\alpha_{x}\right) / \alpha_{x}$. This gapless regime is characterized by a semimetal energy structure with four Weyl cones. The nodes of the Weyl cones appear first on the Fermi surface and move further in the reciprocal space, when the parameters $t_{\mathrm{Z}}^{\perp} / \Delta_{\mathrm{sc}}$ and $\alpha_{y} / \alpha_{x}$ are modified. We also note that if we decrease the ratio $t_{\mathrm{Z}}^{\perp} / \Delta_{\mathrm{sc}}$ up to a point of reaching the phase transition to the trivial phase, the low-energy physics becomes insensitive to the anisotropy.

Floquet Majorana corner states. Next, we analyze the effect of an oscillating in-plane magnetic field that breaks the effective time-reversal symmetry $T_{\text {eff }}$ and, thus, gaps out the helical edge modes of the FHeTS. Nevertheless, the finite system with open boundary conditions along both the $x$ and $y$ axes remains topologically nontrivial as it now hosts a set of zero-energy MCSs, characteristic for the FHOTS phase [96]. The presence of such MCSs is uncovered by focusing on the low-energy degrees of freedom expressed in terms of the Majorana edge modes $\left|\Phi_{ \pm}\right\rangle$. The in-plane Zeeman field $B_{\|}$couples the two helical modes, leading to the following low-energy Hamiltonian density,

$$
\mathcal{H}_{\text {edge }}=\hbar v_{F}^{e}|\boldsymbol{k}| \rho_{z}+\tilde{t}_{\mathrm{Z}}^{\|} \rho_{y},
$$

where the Pauli matrices $\rho_{i}$ act in the space of $\left|\Phi_{ \pm}\right\rangle$, $v_{F}^{e}$ is the velocity of the helical edge modes, and $\tilde{t}_{\mathrm{Z}}^{\|}=t_{\mathrm{Z}}^{\|} \operatorname{Im}\left\langle\Phi_{-}\left|u_{x} \tau_{y} \sigma_{x}+u_{y} \eta_{z} \tau_{y} \sigma_{y}\right| \Phi_{+}\right\rangle$is the "mass term." Thus, our system is described by the well-known JackiwRebbi model [97,98].

Importantly, $\tilde{t}_{\mathrm{Z}}^{\|}$is generally different at different edges. From the symmetry of the modes $\left|\Phi_{ \pm}\right\rangle$, we deduce that the value of the mass term $\tilde{t}_{\mathrm{Z}}^{\|}$is proportional to the projection of the in-plane field $B_{\|}$on the outward-pointing normal unit vector of the corresponding edge in a rectangular geometry (see the SM [95]). Thus, the sign of $\tilde{t}_{\mathrm{Z}}^{\|}$is opposite on two parallel edges at $x=0(y=0)$ and $x=L_{x}\left(y=L_{y}\right)$. Hence, $\tilde{t}_{\mathrm{Z}}^{\|}$has to change its sign at two opposite corners of the 2DEG, leading to the emergence of domain walls at these corners

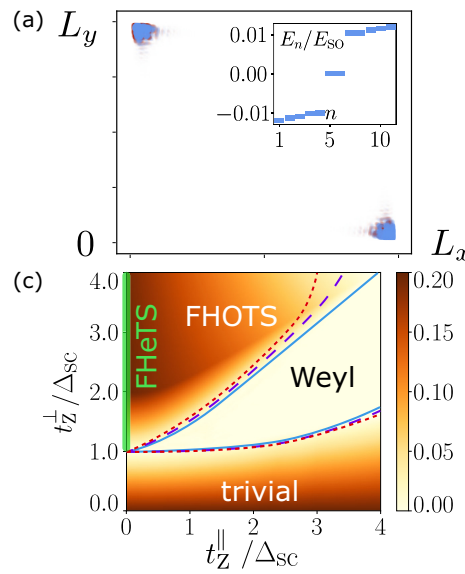

(b)

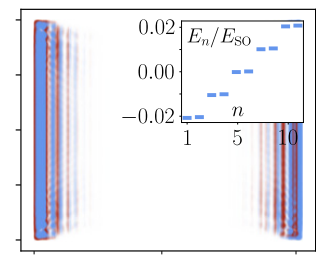

(d)

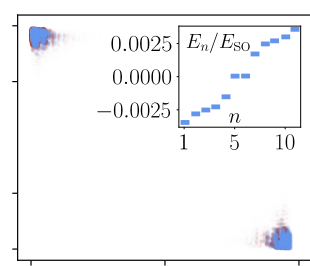

FIG. 3. (a), (b), (d) Probability density of the lowest-energy state in the FHOTS phase for $t_{\mathrm{Z}}^{\perp} / \Delta_{\mathrm{sc}}=3, t_{\mathrm{Z}}^{\|} / \Delta_{\mathrm{sc}}=2.5$, and $\alpha_{x}=\alpha_{y}$. The inset shows the ten lowest eigenenergies. (a) For $\boldsymbol{u}_{\|}=(1,1) / \sqrt{2}$, the in-plane Zeeman field $B_{\|}$opens gaps in all edge modes such that zero-energy MCSs emerge at two opposite corners. (b) The vector $\boldsymbol{u}_{\|}=(0,1)$ is parallel to the edges along which the system stays gapless. (c) Phase diagram showing the bulk gap (color coded in units of $E_{\mathrm{so}}$ ) as a function of the ratios $t_{\mathrm{Z}}^{\perp} / \Delta_{\mathrm{sc}}$ and $t_{\mathrm{Z}}^{\|} / \Delta_{\mathrm{sc}}$ with $\boldsymbol{u}_{\|}=(1,1) / \sqrt{2}$. The critical point $\Delta_{\mathrm{sc}}=t_{\mathrm{Z}}^{\perp}$ at $t_{\mathrm{Z}}^{\|}=0$ merges into a gapless Weyl phase at finite $t_{\mathrm{Z}}^{\|}$. As a result, higher values of $t_{\mathrm{Z}}^{\perp} / \Delta_{\mathrm{sc}}$ are required to reach the topological phase. The various phase boundaries correspond to $\alpha_{y} / \alpha_{x}=0.8$ (red dotted), 0.9 (purple dashed), and 1.0 (blue solid). (d) The MCSs are stable against strong external perturbations and disorder: $\delta \omega=\Delta_{\mathrm{Z}}=0.1 E_{\mathrm{so}}, S_{\mu}=S_{\mathrm{Z}}^{\text {stat }}=$ $S_{\mathrm{Z}}^{\mathrm{dyn}}=0.5 E_{\mathrm{so}}$. A single disorder realization is considered. The rest of the parameters are the same as in Fig. 2.

that host zero-energy MCSs [see Fig. 3(a)]. In the special case when $\boldsymbol{u}_{\|}$is parallel to one of the edges, the corresponding edge modes stay gapless [see Fig. 3(b)].

The simple description in terms of the Jackiw-Rebbi model is expected to work in the regime where the amplitude of the in-plane magnetic field is small. In order to construct the full phase diagram, we calculated numerically the gap to the first excited bulk state as a function of the ratios $t_{\mathrm{Z}}^{\perp} / \Delta_{\mathrm{sc}}$ and $t_{\mathrm{Z}}^{\|} / \Delta_{\mathrm{sc}}$ [see Fig. 3(c)]. The FHOTS phase emerges from the FHeTS phase at nonzero $t_{\mathrm{Z}}^{\|}$. However, if $t_{\mathrm{Z}}^{\|}$is large, the system enters into the gapless Weyl phase. To observe MCSs, the Floquet-Zeeman field perpendicular to the 2DEG plane should dominate such that the condition $t_{\mathrm{Z}}^{\|}<t_{\mathrm{Z}}^{\perp}$ is fulfilled. All the results remain qualitatively the same for a weak anisotropy $\left(\alpha_{x} \neq \alpha_{y}\right)$, which shifts the topological phase transition line only slightly.

Experimental feasibility. Next, we discuss the stability of our setup. We check numerically that the topological phases are robust against an offset $\delta \omega$ in the resonance frequency $\omega$ and a static Zeeman term with strength $\Delta_{Z}$ directed both in plane and out of plane. Similarly, we check the stability with respect to an on-site disorder by adding a fluctuating chemical potential and a static (oscillating) Zeeman field randomly chosen from a uniform distribution with standard deviations $S_{\mu}$ and $S_{\mathrm{Z}}^{\text {stat }}\left(S_{\mathrm{Z}}^{\mathrm{dyn}}\right)$. As a result, we confirm numerically that the MCS are stable against disorder of a strength greatly exceeding the bulk gap [see Fig. 3(d)] (see also the SM [95]). 


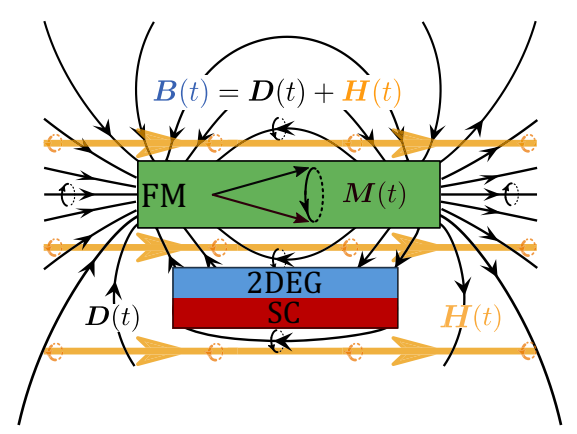

FIG. 4. Setup of the FM layer to generate the desired oscillating magnetic field in the 2DEG. The 2DEG-SC heterostructure is placed in the vicinity of the FM. The system is subjected to an external magnetic field $\boldsymbol{H}(t)=\boldsymbol{H}_{0}+\boldsymbol{h}(t)$ (yellow lines) generating FM resonance (shown not to scale). This induces the precession of the FM magnetization $\boldsymbol{M}(t)$ (black lines inside the FM) and demagnetizing field $\boldsymbol{D}(t)$ (black lines outside of the FM). Close to the surface of the FM layer, the static components of $\boldsymbol{D}(t)$ and $\boldsymbol{H}(t)$ cancel out, so that the dynamics of the total field $\boldsymbol{B}(t)=\boldsymbol{H}(t)+\boldsymbol{D}(t)$ in the 2DEG layer is close to a full $360^{\circ}$ rotation.

The out-of-plane component of the static magnetic field is also not harmful: The MCSs can be observed even for $\Delta_{\mathrm{Z}} \sim t_{\mathrm{Z}}^{\perp}$ and disappear only when the static term becomes stronger.

In experiments, the proximity-induced SC gap $\Delta_{\mathrm{sc}}$ is expected to be of the order of $0.05 \mathrm{meV}$, depending on the properties of the SC and the strength of the coupling between the SC and the 2DEG [99-104]. As shown in this work, the strength of the Floquet-Zeeman amplitude $t_{\mathrm{Z}}^{\perp}$ should exceed $\Delta_{\text {sc }}$ to reach the topological regimes. Hence, assuming that the 2DEG material has an electron $g$-factor $g_{\perp}=15$, the amplitude of the magnetic field $B_{\perp}$ should be of the order of $0.1 \mathrm{~T}$. At the same time, the static component of the magnetic field should be smaller than the dynamic one and the oscillation frequency $\omega$ should be in the $\mathrm{GHz}$ range. The FM layer in the setup is proposed to generate the required Zeeman fields as follows. Applying an external magnetic field $\boldsymbol{H}(t)=\boldsymbol{H}_{0}+\boldsymbol{h}(t)$ with $|\boldsymbol{h}(t)| \ll\left|\boldsymbol{H}_{0}\right|$ and $\boldsymbol{h}(t) \perp \boldsymbol{H}_{0}$ under FM resonance condition induces a precession of the FM magnetization $\boldsymbol{M}(t)[105,106]$. The precession cone of $\boldsymbol{M}(t)$ depends on the angle between the FM easy axis and $\boldsymbol{H}_{0}$, while the resonance frequency is determined by the magnitude $\left|\boldsymbol{H}_{0}\right|$. Outside of the FM, the total field $\boldsymbol{B}(t)$ is equal to the sum of the external field $\boldsymbol{H}(t)$ and an oscillating demagnetizing field $\boldsymbol{D}(t)$ (see Fig. 4). Hence, by carefully choosing the system geometry, the static component of $\boldsymbol{B}(t)$ could be adjusted close to zero over a large region of space in the proximity of the FM surface including the $2 \mathrm{DEG}$. The amplitude of the remaining oscillating component overcomes the threshold of $0.1 \mathrm{~T}$ inside the 2DEG, as we have confirmed by micromagnetic simulations (see the SM [95]). It is important that the generated magnetic field is very small and below the critical field of the superconductor to avoid the appearance of vortices or domain walls. Promising candidates for such FMs are, e.g., EuS [65], GdN [66], and yttrium iron garnet (YIG) [67].

Alternatively, the fast switching or the sustained oscillation of the FM magnetization has already been achieved experimentally by shining optical light on a FM (via an all-optical magnetization reversal) [107-109], by applying piezostrain [110,111], or by injecting a spin-polarized current (via a spinorbit torque) [112-116]. This domain of research is currently under an active exploration because of its crucial role in the implementation of magnetic memory and logic devices. We also note that in our setup, the magnetic field in the 2DEG can originate from both the FM demagnetizing field at a moderate range and from the exchange interactions at atomic distances.

Conclusions. We have considered a Floquet triple-layer setup of a 2DEG proximity coupled to a SC and a FM. Under resonant drive the FM induces an oscillating Zeeman field in the 2DEG. The out-of-plane component of the magnetic field competes with the proximity-induced SC gap and leads to the emergence of the FHeTS hosting an effective Kramers pair of gapless helical edge modes. Moreover, the in-plane component of the magnetic field enters into the low-energy description corresponding to the effective JackiwRebbi model as a mass term and opens a gap in the edge mode spectrum. Change in the sign of the mass term, which inevitably occurs at two opposite corners of the system in a rectangular geometry, leads to the emergence of Floquet MCSs. We argued that the proposed setup is within experimental reach combining available magnetic, semiconducting, and superconducting materials.

Acknowledgments. We acknowledge very much the discussions with Patrick Maletinsky, Martino Poggio, Christina Psaroudaki, Marko Rančić, and Flavio Ronetti. This work was supported by the Swiss National Science Foundation, NCCR QSIT, and the Georg H. Endress Foundation. This project received funding from the European Union's Horizon 2020 research and innovation program (ERC Starting Grant, Grant Agreement No. 757725).
[1] M. Z. Hasan and C. L. Kane, Rev. Mod. Phys. 82, 3045 (2010).

[2] X.-L. Qi and S.-C. Zhang, Rev. Mod. Phys. 83, 1057 (2011).

[3] M. Sato and Y. Ando, Rep. Prog. Phys. 80, 076501 (2017).

[4] J. Wang and S.-C. Zhang, Nat. Mater. 16, 1062 (2017).

[5] X.-G. Wen, Rev. Mod. Phys. 89, 041004 (2017).

[6] W. A. Benalcazar, B. A. Bernevig, and T. L. Hughes, Science 357, 61 (2017).

[7] W. A. Benalcazar, B. A. Bernevig, and T. L. Hughes, Phys. Rev. B 96, 245115 (2017).
[8] Z. Song, Z. Fang, and C. Fang, Phys. Rev. Lett. 119, 246402 (2017).

[9] Y. Peng, Y. Bao, and F. von Oppen, Phys. Rev. B 95, 235143 (2017).

[10] S. Imhof, C. Berger, F. Bayer, J. Brehm, L. W. Molenkamp, T. Kiessling, F. Schindler, C. Hua Lee, M. Greiter, T. Neupert, and R. Thomale, Nat. Phys. 14, 925 (2018).

[11] M. Geier, L. Trifunovic, M. Hoskam, and P. W. Brouwer, Phys. Rev. B 97, 205135 (2018). 
[12] F. Schindler, A. M. Cook, M. G. Vergniory, Z. Wang, S. S. P. Parkin, B. A. Bernevig, and T. Neupert, Sci. Adv. 4, eaat0346 (2018).

[13] C.-H. Hsu, P. Stano, J. Klinovaja, and D. Loss, Phys. Rev. Lett. 121, 196801 (2018).

[14] T. Liu, J. J. He, and F. Nori, Phys. Rev. B 98, 245413 (2018).

[15] Y. Volpez, D. Loss, and J. Klinovaja, Phys. Rev. Lett. 122, 126402 (2019).

[16] M. Ezawa, Sci. Rep. 9, 5286 (2019).

[17] A. Agarwala, V. Juricic, and B. Roy, arXiv:1902.00507.

[18] K. Laubscher, D. Loss, and J. Klinovaja, arXiv:1905.00885.

[19] D. Calugaru, V. Juricic, and B. Roy, Phys. Rev. B 99, 041301(R) (2019).

[20] S. Franca, J. van den Brink, and I. C. Fulga, Phys. Rev. B 98, 201114(R) (2018).

[21] R. W. Bomantara, L. Zhou, J. Pan, and J. Gong, Phys. Rev. B 99, 045441 (2019).

[22] Y. Peng and G. Refael, Phys. Rev. Lett. 123, 016806 (2019).

[23] B. Huang and W. V. Liu, arXiv:1811.00555.

[24] M. Rodriguez-Vega, A. Kumar, and B. Seradjeh, Phys. Rev. B 100, 085138 (2019).

[25] R. Seshadri, A. Dutta, and D. Sen, Phys. Rev. B 100, 115403 (2019).

[26] T. Nag, V. Juricic, and B. Roy, arXiv:1904.07247.

[27] H. Hu, B. Huang, E. Zhao, and W. V. Liu, arXiv:1905.03727.

[28] J. H. Shirley, Phys. Rev. 138, B979 (1965).

[29] N. Goldman and J. Dalibard, Phys. Rev. X 4, 031027 (2014).

[30] M. Bukov, L. D’Alessio, and A. Polkovnikov, Adv. Phys. 64, 139 (2015)

[31] A. Eckardt and E. Anisimovas, New. J. Phys. 17, 093039 (2015).

[32] T. Oka and H. Aoki, Phys. Rev. B 79, 081406(R) (2009).

[33] J.-I. Inoue and A. Tanaka, Phys. Rev. Lett. 105, 017401 (2010).

[34] T. Kitagawa, T. Oka, A. Brataas, L. Fu, and E. Demler, Phys. Rev. B 84, 235108 (2011).

[35] N. H. Lindner, G. Refael, and V. Galitski, Nat. Phys. 7, 490 (2011).

[36] N. H. Lindner, D. L. Bergman, G. Refael, and V. Galitski, Phys. Rev. B 87, 235131 (2013).

[37] J. Cayssol, B. Dóra, F. Simon, and R. Moessner, Phys. Status Solidi RRL 7, 101 (2013).

[38] P. M. Perez-Piskunow, G. Usaj, C. A. Balseiro, and L. E. F. Foa Torres, Phys. Rev. B 89, 121401(R) (2014).

[39] J. Klinovaja, P. Stano, and D. Loss, Phys. Rev. Lett. 116, 176401 (2016)

[40] T. Kitagawa, M. A. Broome, A. Fedrizzi, M. S. Rudner, E. Berg, I. Kassal, A. Aspuru-Guzik, E. Demler, and A. G. White, Nat. Commun. 3, 882 (2012).

[41] M. C. Rechtsman, J. M. Zeuner, Y. Plotnik, Y. Lumer, D. Podolsky, F. Dreisow, S. Nolte, M. Segev, and A. Szameit, Nature (London) 496, 196 (2013).

[42] M. Aidelsburger, M. Atala, M. Lohse, J. T. Barreiro, B. Paredes, and I. Bloch, Phys. Rev. Lett. 111, 185301 (2013).

[43] G. Jotzu, M. Messer, R. Desbuquois, M. Lebrat, T. Uehlinger, D. Greif, and T. Esslinger, Nature (London) 515, 237 (2014).

[44] K. Le Hur, L. Henriet, A. Petrescu, K. Plekhanov, G. Roux, and M. Schiró, C. R. Phys. 17, 808 (2016).

[45] T. Kitagawa, E. Berg, M. Rudner, and E. Demler, Phys. Rev. B 82, 235114 (2010).
[46] M. S. Rudner, N. H. Lindner, E. Berg, and M. Levin, Phys. Rev. X 3, 031005 (2013).

[47] R. Roy and F. Harper, Phys. Rev. B 96, 155118 (2017).

[48] L. J. Maczewsky, J. M. Zeuner, S. Nolte, and A. Szameit, Nat. Commun. 8, 13756 (2017).

[49] S. Yao, Z. Yan, and Z. Wang, Phys. Rev. B 96, 195303 (2017).

[50] G. M. Graf and C. Tauber, Ann. Henri Poincaré 19, 709 (2018).

[51] A. G. Grushin, Á. Gómez-León, and T. Neupert, Phys. Rev. Lett. 112, 156801 (2014).

[52] L. Jiang, T. Kitagawa, J. Alicea, A. R. Akhmerov, D. Pekker, G. Refael, J. I. Cirac, E. Demler, M. D. Lukin, and P. Zoller, Phys. Rev. Lett. 106, 220402 (2011).

[53] A. A. Reynoso and D. Frustaglia, Phys. Rev. B 87, 115420 (2013).

[54] M. Thakurathi, A. A. Patel, D. Sen, and A. Dutta, Phys. Rev. B 88, 155133 (2013).

[55] D. E. Liu, A. Levchenko, and H. U. Baranger, Phys. Rev. Lett. 111, 047002 (2013).

[56] A. Kundu and B. Seradjeh, Phys. Rev. Lett. 111, 136402 (2013).

[57] M. Thakurathi, K. Sengupta, and D. Sen, Phys. Rev. B 89 , 235434 (2014).

[58] M. Thakurathi, D. Loss, and J. Klinovaja, Phys. Rev. B 95, 155407 (2017).

[59] D. M. Kennes, N. Müller, M. Pletyukhov, C. Weber, C. Bruder, F. Hassler, J. Klinovaja, D. Loss, and H. Schoeller, Phys. Rev. B 100, 041103 (2019).

[60] D. T. Liu, J. Shabani, and A. Mitra, Phys. Rev. B 99, 094303 (2019).

[61] E. I. Rashba, Sov. Phys. Solid State 2, 1109 (1960).

[62] C. Kloeffel and D. Loss, Annu. Rev. Condens. Matter Phys. 4, 51 (2013).

[63] A. I. Buzdin, Rev. Mod. Phys. 77, 935 (2005).

[64] B. Li, N. Roschewsky, B. A. Assaf, M. Eich, M. EpsteinMartin, D. Heiman, M. Münzenberg, and J. S. Moodera, Phys. Rev. Lett. 110, 097001 (2013).

[65] F. Katmis, V. Lauter, F. S. Nogueira, B. A. Assaf, M. E. Jamer, P. Wei, B. Satpati, J. W. F. I. Eremin, D. Heiman, P. JarilloHerrero, and J. S. Moodera, Nature (London) 533, 513 (2016).

[66] A. Kandala, A. Richardella, D. W. Rench, D. M. Zhang, T. C. Flanagan, and N. Samarth, Appl. Phys. Lett. 103, 202409 (2013).

[67] Z. Jiang, C.-Z. Chang, C. Tang, J.-G. Zheng, J. S. Moodera, and J. Shi, AIP Adv. 6, 055809 (2016).

[68] J. Nitta, T. Akazaki, H. Takayanagi, and T. Enoki, Phys. Rev. Lett. 78, 1335 (1997).

[69] G. Engels, J. Lange, T. Schapers, and H. Luth, Phys. Rev. B 55, R1958(R) (1997).

[70] J. Schliemann, J. C. Egues, and D. Loss, Phys. Rev. Lett. 90, 146801 (2003).

[71] B. A. Bernevig, J. Orenstein, and S. C. Zhang, Phys. Rev. Lett. 97, 236601 (2006).

[72] R. Winkler, Spin-Orbit Coupling Effects in Two-Dimensional Electron and Hole Systems (Springer, Berlin, 2003).

[73] J. D. Koralek, C. P. Weber, J. Orenstein, B. A. Bernevig, S.-C. Zhang, S. Mack, and D. D. Awschalom, Nature (London) 458, 610 (2009).

[74] M. Duckheim, D. L. Maslov, and D. Loss, Phys. Rev. B 80, 235327 (2009). 
[75] T. Meng, J. Klinovaja, and D. Loss, Phys. Rev. B 89, 205133 (2014).

[76] C. L. Kane, R. Mukhopadhyay, and T. C. Lubensky, Phys. Rev. Lett. 88, 036401 (2002).

[77] D. Poilblanc, G. Montambaux, M. Héritier, and P. Lederer, Phys. Rev. Lett. 58, 270 (1987).

[78] L. P. Gor'kov and A. G. Lebed, Phys. Rev. B 51, 3285 (1995).

[79] J. Klinovaja and D. Loss, Phys. Rev. Lett. 111, 196401 (2013).

[80] J. C. Y. Teo and C. L. Kane, Phys. Rev. B 89, 085101 (2014).

[81] J. Klinovaja and D. Loss, Eur. Phys. J. B 87, 171 (2014).

[82] T. Meng, P. Stano, J. Klinovaja, and D. Loss, Eur. Phys. J. B 87, 203 (2014).

[83] T. Neupert, C. Chamon, C. Mudry, and R. Thomale, Phys. Rev. B 90, 205101 (2014).

[84] E. Sagi and Y. Oreg, Phys. Rev. B 90, 201102(R) (2014).

[85] J. Klinovaja, Y. Tserkovnyak, and D. Loss, Phys. Rev. B 91, 085426 (2015).

[86] R. A. Santos, C.-W. Huang, Y. Gefen, and D. B. Gutman, Phys. Rev. B 91, 205141 (2015).

[87] P.-H. Huang, J.-H. Chen, P. R. S. Gomes, T. Neupert, C. Chamon, and C. Mudry, Phys. Rev. B 93, 205123 (2016).

[88] P. Le Jeune, D. Robart, X. Marie, T. Amand, M. Brosseau, J. Barrau, and V. Kalevcih, Semicond. Sci. Technol. 12, 380 (1997).

[89] A. Malinowski and R. T. Harley, Phys. Rev. B 62, 2051 (2000).

[90] M. A. Toloza Sandoval, A. Ferreira da Silva, E. A. de Andrada e Silva, and G. C. La Rocca, Phys. Rev. B 86, 195302 (2012).

[91] V. Mourik, K. Zuo, S. M. Frolov, S. R. Plissard, E. P. A. M. Bakkers, and L. P. Kouwenhoven, Science 336, 1003 (2012).

[92] M. T. Deng, C. L. Yu, G. Y. Huang, M. Larsson, P. Caroff, and H. Q. Xu, Nano Lett. 12, 6414 (2012).

[93] R. M. Lutchyn, J. D. Sau, and S. Das Sarma, Phys. Rev. Lett. 105, 077001 (2010).

[94] Y. Oreg, G. Refael, and F. von Oppen, Phys. Rev. Lett. 105, 177002 (2010).

[95] See Supplemental Material at http://link.aps.org/supplemental/ 10.1103/PhysRevResearch.1.032013 for (i) details of derivation of the topological criterion; (ii) an analytical description of helical edge modes and Majorana corner states; (iii) numerical results in a geometry with modified boundary conditions; (iv) a study of the role of anisotropy on the topological phase diagram; (v) an explicit form of the discretized Hamiltonian used in numerical calculations; (vi) micromagnetic simulations of the magnetization dynamics in the driven triple-layer setup; and (vii) the stability check of Majorana corner states for various strength of the external perturbations and disorder.

[96] The initial presence of the effective symmerty $T_{\text {eff }}$ is not crucial for our setup, as this symmetry is broken by the in-plane field anyway, thus, our setup is stable against disorder and does not rely on the presence of particular spatial symmetries.

[97] R. Jackiw and C. Rebbi, Phys. Rev. D 13, 3398 (1976).

[98] R. Jackiw and J. Schrieffer, Nucl. Phys. B 190, 253 (1981).

[99] W. Chang, S. M. Albrecht, T. S. Jespersen, F. Kuemmeth, P. Krogstrup, J. Nygard, and C. M. Marcus, Nat. Nanotechnol. 10, 232 (2015).

[100] Z. Wan, A. Kazakov, M. J. Manfra, L. N. Pfeiffer, K. W. West, and L. P. Rokhinson, Nat. Commun. 6, 7426 (2015).

[101] M. Kjaergaard, F. Nichele, H. J. Suominen, M. P. Nowak, M. Wimmer, A. R. Akhmerov, J. A. Folk, K. Flensberg, J. Shabani, C. J. Palmstrøm, and C. M. Marcus, Nat. Commun. 7, 12841 (2016).

[102] J. Shabani, M. Kjaergaard, H. J. Suominen, Y. Kim, F. Nichele, K. Pakrouski, T. Stankevic, R. M. Lutchyn, P. Krogstrup, R. Feidenhansl, S. Kraemer, C. Nayak, M. Troyer, C. M. Marcus, and C. J. Palmstrom, Phys. Rev. B 93, 155402 (2016).

[103] C. Reeg, D. Loss, and J. Klinovaja, Beilstein J. Nanotechnol. 9, 1263 (2018).

[104] H. Ren, F. Pientka, S. Hart, A. Pierce, M. Kosowsky, L. Lunczer, R. Schlereth, B. Scharf, E. M. Hankiewicz, L. W. Molenkamp, B. I. Halperin, and A. Yacoby, Nature (London) 569, 93 (2019).

[105] C. Kittel, Phys. Rev. 73, 155 (1948).

[106] C. Kittel, J. Phys. Radium 12, 291 (1951).

[107] C. D. Stanciu, F. Hansteen, A. V. Kimel, A. Kirilyuk, A. Tsukamoto, A. Itoh, and T. Rasing, Phys. Rev. Lett. 99, 047601 (2007).

[108] A. Kirilyuk, A. V. Kimel, and T. Rasing, Rev. Mod. Phys. 82, 2731 (2010)

[109] S. Higashikawa, H. Fujita, and M. Sato, arXiv:1810.01103.

[110] R.-C. Peng, J.-M. Hu, K. Momeni, J.-J. Wang, L.-Q. Chen, and C.-W. Nan, Sci. Rep. 6, 27561 (2016).

[111] Q. Wang, J. Domann, G. Yu, A. Barra, K. L. Wang, and G. P. Carman, Phys. Rev. Applied 10, 034052 (2018).

[112] I. M. Miron, K. Garello, G. Gaudin, P.-J. Zermatten, M. V. Costache, S. Auffret, S. Bandiera, B. Rodmacq, A. Schuhl, and P. Gambardella, Nature (London) 476, 189 (2011).

[113] L. Liu, C.-F. Pai, Y. Li, H. W. Tseng, D. C. Ralph, and R. A. Buhrman, Science 336, 555 (2012).

[114] P. Gambardella and I. M. Miron, Philos. Trans. R. Soc., A 369, 3175 (2011)

[115] J. Sinova, S. O. Valenzuela, J. Wunderlich, C. H. Back, and T. Jungwirth, Rev. Mod. Phys. 87, 1213 (2015).

[116] A. Manchon, J. Zelezný, I. M. Miron, T. Jungwirth, J. Sinova, A. Thiaville, K. Garello, and P. Gambardella, Rev. Mod. Phys. 91, 035004 (2019) 